\title{
A cane-based low cost sensor to implement attention mechanisms in telecare robots
}

\author{
Joaquin Ballesteros $^{1}$, Alberto Tudela ${ }^{2}$, Juan Rafael Caro-Romero ${ }^{2}$ and Cristina Urdiales ${ }^{2}$
}

\begin{abstract}
Telepresence robots have been recently used for Comprehensive Geriatric Assessment (CGA). Since the robot can not track a person continuously, there are several strategies to decide when to check them, from cyclic checks to simple requests from users and/or caregivers. In order to adapt to the user needs and condition, it is preferable to perform CGA as soon as regularities appear. However, this requires detection of potential issues in users to offer immediate service. In this work we propose a new low cost force sensor system to detect user's condition and attract attention of CGA robots, so they can perform a full examination on a need basis. The main advantages of this system are: i) it can be attached to any standard commercial cane; ii) its power consumption is very reduced; and iii) it provides continuous information as long as the user walks. It has been tested with several elderly volunteers in care facilities. Results have proven that the sensor readings are indeed correlated with the users' condition.
\end{abstract}

\section{INTRODUCTION}

Telepresence robots have been traditionally used to allow elderly people to remain in their homes for longer and to stay connected to their friends and family. Recently, these robots can also be useful for home care assistance and/or Comprehensive Geriatric Assessment (CGA) [1], [2], [3]. Equipping a full house with the sensors and actuators required in assistive tasks is costly, complex, and attached to a specific location. Robots bring on-board the required sensors and actuators wherever they are needed. However, robots are not meant to track a person continuously. Furthermore, due to their cost, these robots are often used in care institutions where they are meant to assist several persons on a daily basis. There are several strategies to decide when to find and assist a person, from cyclic checks to simple requests from users and/or caregivers. However, in order to work preventively, it would be helpful to go to a person as soon as irregularities are expected. To avoid fixed installations, these irregularities could be detected by simple, wearable sensors in the users. Although these sensors are not as reliable nor accurate as the robot on-board sensors, they could demand its attention to the person location and trigger CGA and/or any

\footnotetext{
*This work has been partly supported by Plan Propio de la Universidad de Malaga (Proyectos Puente) at Malaga University, and Swedish Knowledge Foundation (KKS) through the research profile Embedded Sensor Systems for Health at Mälardalen University, Sweden. Also, author would like to acknowledge Ms. Luna Ruiz for her support gathering information from users.

${ }^{1}$ Joaquin Ballesteros is with Division of Intelligent $\mathrm{Fu}$ ture Technologies, Mälardalen University, Västerås, Sweden joaquin.ballesteros@mdh. se

${ }^{2}$ Alberto Tudela, Juan Rafael Caro-Romero and Cristina Urdiales are with Department of Electronic Technology, University of Malaga, Malaga, Spain ajtudela@uma.es jrcaro@uma.es acurdiales@uma.es
}

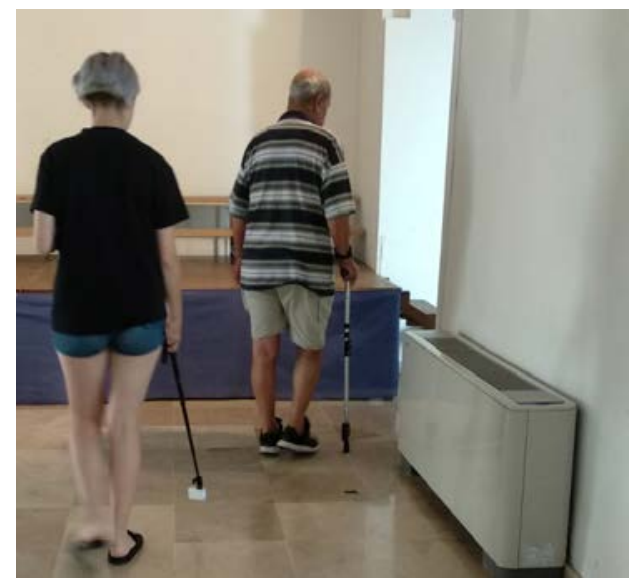

Fig. 1. Cane user walking during a free walking test.

available kind of assistance. However, wearable sensors often need to be calibrated and/or located in specific body parts, requiring an extra effort from the users [4], who may forget to wear them for long-term monitoring periods and/or on a daily basis. An alternative is to attach sensors to elements that people use on a daily basis.

Currently in United States of America, one out of four seniors need a mobility platform to walk [5]. Canes are the most common mobility platform $(16.4 \%$ of the total population in United States of America) [5]. Hence, attaching sensors to a standard cane may solve the problems presented before, as users can not forget to use their mobility platforms because they need it to walk. Furthermore, when cane sensors are not providing information, it means that users are not walking. Changes in activity periods also provide valuable information on the person's condition. Cane sensors may provide information on two relevant health related parameters: weight bearing and gait trends [6], [7].

Wearable sensors like footwear-based systems [8] can also be used to measure load on a cane, but, as commented, they often need to be calibrated and/or located in specific body parts, requiring an extra effort from the users [4]. Alternatively, the authors have already proposed to add sensors to walk assistive devices like a rollator [9], [10]. Although these devices often have lower accuracy when compared to other solutions because they measure users' effects on the mobility platform [11], they are fit to detect changes in how much load does the user supports on their affected side. This is an indirect measure on their condition and it can be used to monitor their condition [12].

This work proposes a low cost sensor system for long-term 
monitoring of cane load measurements, i.e. user's condition (Figure 1). Significant changes in cane loads are transmitted via BT to external systems, so telecare robots can be warned on a need basis to provide assistance or a more reliable assessment with their on-board sensors. The main goals of the proposed system are: i) it is totally transparent to the user; ii) compatibility with existing commercial canes; iii) no impact on cane ergonomics; and iv) low power consumption for continuous, long term use. Additionally, the proposed system will be released under a Creative Commons License to enhance its reach and impact.

The system has been tested by 8 volunteering elderly cane users in two Senior Care facilities. Results prove that our system successfully measures the cane loads in different environments during free walking tests. We have also checked that load bearing is correlated with user's condition, which has been evaluated using the well known 10 Meters Test [13]. Section 2 describes the mechanical design, electronics and testing of the proposed system. Section 3 presents our load cane estimation algorithm. Section 4 describes our experiments and results. Section 5 discusses the results and present the future work.

\section{CANE ADAPTATION}

On-board sensors on most smart canes are located either on the handgrip [6], [14], shaft [15], [16], [17], [7], [14], [7] or tip [6], [15]. Placing sensors on the handgrip or tip may involve major cane modifications [6], [14]. As both locations affect how users support their weight, these modifications must be ergonomic. The shaft allows more space to place the electronic. However, this approach may involve changes in the cane center of gravity and also, in its weight [16], [15], [7], [14]. Even in a best case scenario, any modification significantly affecting a walking aid requires an extensively validation and/or certification process. In this work, the system has been designed to be inserted into a standard commercial change without any significant modification, so people can use it transparently in their own aids. This section describes the mechanical design and electronic components to achieve this goal.

1) Cane mechanical design: Our design includes two different elements: sensors and microcontrollers. The sensors have been embedded in the closest part of the shaft respect to the tip (Figure 2(a)). Thus, the tip and handgrip designs are not modified and we preserve the cane original ergonomics properties. Besides, we have attached electronics to shaft electronic in a location where changes in the center of gravity are minimized.

Figure 2 shows how this system involves minor cane modifications. Installation simply consists of removing the cane tip to insert the designed sensor module, which is fully described in next section. The shaft requires a small perforation to connect this module to a external attached microntroller. Each piece has been modelled using Autodesk $\mathbb{R}$ Fusion 360 and it has been 3D printed with Ultimaker2 using PLA plastic. They are fully compatible with standard commercial canes. Since cane shafts present different diameters,

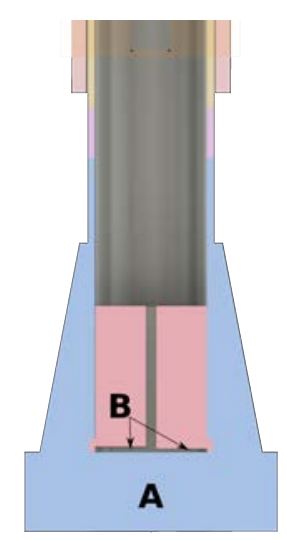

(a) Sensors Area

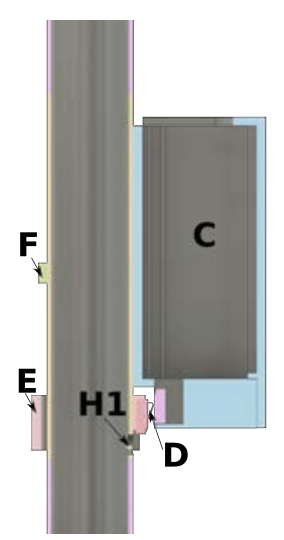

(b) MCU area
Fig. 2. Mechanical design parts: A) rubber tip; B) force sensors locations; C) microcontroller (MCU) and battery box; D) connectors between sensors and MCU; E) connector ring and F) clamp for MCU box.

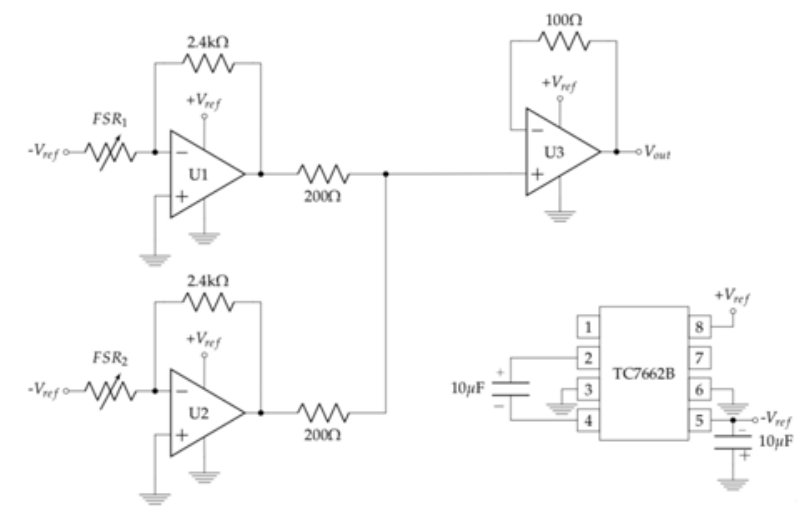

Fig. 3. Sensor electronic board. The input signals to FS402 sensors are inverted. $U 1, U 2$ filter the force sensors outputs. Then, $U 3$ sums both signal to obtain the output.

pieces have been designed to be easily scalable to fit any over $17 \mathrm{~mm}$. All 3D models are freely available online at https://github.com/joaquinballesteros/Smart-Cane.

2) Electronic design: There are different options to measure the load on the cane, both in handgrips and shafts [6], [14][7]. The main drawback of most existing approaches is that they rely on relatively expensive electronic components, such as piezoelectric quartz force link with in-line amplifier (Kistler Instrument Corp., Novi, MI) [7] or array of force sensor (FSR 402, Interlink Electronics, USA) [6], reducing affordability for end-users. The main drawback of simpler, cheaper sensors is that their range is very limited, usually $10 \mathrm{Kg}$ or less. This problem can be partially mitigated in medium/large surfaces, where several cheap sensors can be strategically distributed to measure partial loads on different areas of the surface and their readings can be processed and combined. However, the contact surface of a cane is typically too small to allocate more than two sensors, plus weight is not uniformly distributed during the gait cycle. Unfortunately, elderly people with disabilities tend to support a significant percentage of their weight on their walking aids, so this range imposes a severe constraint. 
In our case, in order to overcome this limitation, our electronic system relies on two force sensors embedded inside the shaft at different depths (B in figure 2(a)) to significantly increase the measuring range (see next section). We have designed a specific 3D printer plastic piece to fit them perfectly inside the shaft. This approach is affordable because it only involves: two low cost sensors (FSR 402, Interlink Electronics, USA), an inverter (TC7662B, Microchip Technology, USA), an array of operational amplifiers (OPA347, Texas Instruments, USA) and some resistors and capacitors. Figure 3 shows the sensor board. The input signals to FS402 are inverted $-V_{\text {ref }}$ using TC7662B circuit. The operational amplifiers $\mathrm{U} 1, \mathrm{U} 2$ and $\mathrm{U} 3$ are adjusted with a $2.4 \mathrm{k} \Omega$ and $100 \Omega$ resistors respectively to obtain the higher range of support measurement. The filtered output of FSR 402 force sensors U1, U2 are added to obtain the combination of both sensors U3 via hardware. The sensor board provides a $50 \mathrm{~Hz}$ output.

In our current implementation we are working with a BLE nano v2 microcontroller (nrf51822, Nordic Semiconductor, Norway). It transmits packages of 20 Bytes (8 readings) at $6.25 \mathrm{~Hz}$ to any paired device. Long-term monitoring requires a working plan for at least 12 hours without recharging. For this reason, we have chosen a $1 \mathrm{~S} 1 \mathrm{P} 500 \mathrm{mAh}$ Lipo Battery that we have heuristically checked that can be used over 4 days of use without recharging.

As a whole, the cost of the whole system is cheap when compared to other solutions -less than USD 100 in total-, plus it requires only minor modifications in the cane.

\section{DYNAMIC WEIGHT BEARING CALCULATION}

As commented, the main challenge in our sensor system is how to extend the reading range of low cost sensors. Analysing gait, we can obtain a rough estimation of how much weight users bear on their walking aid. Cane users can be categorised into contralateral and ipsilateral. Users who support weight on the cane and on the closest foot to the cane at the same time are ipsilateral. The others are contralateral. Ipsilateral users reportedly load up to $7 \%$ of their body weight on the cane. Contralateral users are more frequent and also more critical, as they load up to 9\% [18]. These percentages may increase when users have some physical issues. FSR 402 sensors have a limited range up to only $10 \mathrm{~kg}$. Therefore, contralateral, otherwise healthy users could weight as much as $111 \mathrm{Kg}^{1}$ before sensors saturate. Unfortunately, this limit could be significantly more restricting for users with physical issues.

In order to increase the measurement range while keeping sensors affordable, we have placed two ${ }^{2}$ FSR 402 sensors at different depths on a circular 3D printed plastic piece. First, weight is distributed all over the surface of the piece (Fig.4(a)), so each sensor only receives part of the load,

\footnotetext{
${ }^{1}$ Contralateral users support up to $9 \%$ and the maximum detection is $10 \mathrm{Kg}$, hence $\frac{10 \mathrm{~kg}}{0.09} \simeq 111 \mathrm{Kg}$ is the maximum load detected on each FSR 402 sensor.

${ }^{2}$ Due to the limit in the inner cane diameter (maximum of $22 \mathrm{~mm}$ ), only two force sensors can be placed.
}

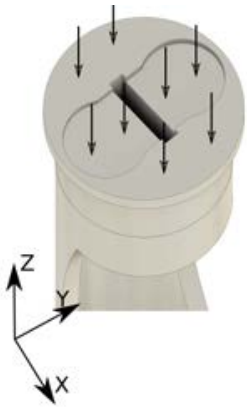

(a)

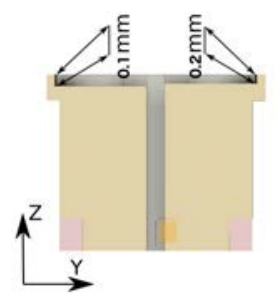

(b)
Fig. 4. Pressure distribution: (a) Rubber tip pressure distribution among the force sensors and the sensor area; (b) Pressure distribution on sensors depending on depth

effectively increasing the global piece range. Additionally, the tip rubber applies different pressure on each sensor (Figure 4(b)), i.e. lower loads will not affect the deepest sensor. Unfortunately, factors like the nature of materials, non-linearity in sensors and other physical variables, it is very hard to analytically calculate the sensor area output function. Hence, we have calibrated the system through extensive testing. After applying known different static weights on the fully vertical cane, the following approximation to load on cane has been obtained: $f(x)=2.5193 .10^{8} x^{3}-$ $2.4351 .10^{5} x^{2}+2.7607 .10^{2} x-0.4271 \mathrm{x}$ being the sensor reading.

The designed 2-sensors piece output range grows up to $30 \mathrm{Kg}$ (hardware reading equal to 1024) for the best depth difference. This means that a healthy contralateral user could weight as much as $333 \mathrm{Kg}$. Even though persons with disabilities support significantly more than $9 \%$ on the cane, this limit is high enough for most cases.

While users walk, the weight they bear on the cane keeps changing. In order to calculate the maximum support that a given user needs while walking, gait cycles can be analysed using the adapted cane. Figure 6(d) shows the typical cane movements for contralateral users while they walk. During a gait cycle, maximum force is applied on the cane when it is fully vertical, as the load vector is orthogonal to the force sensors plane. The cane support cycle corresponds to the elapsed time from a heel strike of the opposite leg with respect to the cane- to the next one. As expected, force sensors outputs fluctuate while users change their load on the cane (Figure 5(b)). As commented, maximum peak values correspond to the cane in a vertical position. A peak during a cane support period represents the upper bound support that a given user needs in each step. A sequence of peaks in time provides continuous information about the user load on the cane. We have used the findpeaks MATLAB R2016b (The MathWorks, Inc., Natick, Massachusetts, United States) function to detect all peaks during a given test. The function has been set with a MinPeakDistance parameter equal to 25, as the minimum time between two cane support is 0.5 . This value is valid for more than $99,99 \%$ of the elderly population [19]. The function second parameter, MinPeakHeight is used 
to filter out spurious peaks from the input signal. Its value depends on users' load, so we have empirically set it to the average of the input signal exercise.

\section{EXPERIMENT AND RESULTS}

In order to check if our device can detect anomalous trends in cane users to potentially attract the attention of a telecare robot, we need to test it with the target population. Assistive devices are often tested with healthy volunteers, but it has been consistently reported that the behaviour of people with disabilities is hard to emulate after a while. Specifically, people who do not require a cane to walk support their weight on them in a different, non consistent way. Anomalous trends can be detected either by working with one user for a extended time period or with several ones with different conditions in limited time periods. The second approach is typically easier due to time constraints and also because it is possible to assess the users' conditions beforehand with clinician tests and/or scale and check whether results are consistent or not.

In our case, we have tested a modified cane with volunteers who usually require a traditional cane for mobility in their Activities of Daily Living. Tests were carried out in two senior centres in Cordoba, Spain.

Volunteers were asked to complete two different tests sequentially. First, they performed the 10 Meters Test while a mobile phone gather the information from the cane via BT (Figure 1). We used the 10 Meters Test to assess the users' condition for benchmarking. Then, the were asked to keep walking for at least 1 more minute. Our tests were approved by the University of Malaga Institutional Ethical Committee. Additionally, tests were approved by the senior centres and all volunteers signed an informed consent.

In this work, we have analysed data from 8 volunteers: 6 men and 2 women. Participants were in average $82.13 \pm$ 5.99 years old (range 74-91 years). Table I shows their age, gender, average gait speed -obtained from the 10 Meters Testand their reported physical disabilities.

It can be observed that some volunteers present less severe disabilities than others. Reportedly, walking speed is related to user's condition: the faster they walk, the less severe their walking disability is. People with severe dependencies typically present gait speeds below $0.6 \mathrm{~m} / \mathrm{s}$ [13]. For example, volunteers 7 and 8 are clearly less affected by their disability than volunteer 4 .

We also measured that some users load significantly more weight on the cane with respect to others. For example, user 4 (Figure 6 .a) presents peak values of $11.78 \mathrm{Kg}$ in average when compared to user like $5-0.32 \mathrm{Kg}$ - (Figure 6 .c). The main reason for this variability is that load depends largely on the users' condition, even more than on the users' weight, i.e., users with poor condition usually need more assistance. Indeed, some volunteers like user 5 simply rely on the cane occasionally for balance. It can be observed in Figure 6 .d that at some time instants, the cane is simply dragger around. On the contrary, it can be observed in Figure 6 .b that volunteer 4 heavily leans on the cane when he has to support his weight on the affected side.

Figure 7 shows the relationship between gait speed during our 10 meter tests and the load peaks for each of our volunteers. As expected, gait speeds below $0.615 \mathrm{~m} / \mathrm{s}$ are related with higher loads on cane: $5.95 \mathrm{Kg}$ in average, ranging from $1.15 \mathrm{Kg}$ to $11.78 \mathrm{Kg}$ ), whereas volunteers with gait speeds above its $0.63 \mathrm{Kg}$ loads range from $0.18 \mathrm{Kg}$ to $1.45 \mathrm{Kg}$. Additionally, load variances for gait speeds under $0.615 \mathrm{~m} / \mathrm{s}$ (variation range from $0.84 \mathrm{Kg}$ to $2.69 \mathrm{Kg}$ ) are higher when compared to gait speeds above this limit (variation range from $0.17 \mathrm{Kg}$ to $0.68 \mathrm{Kg}$ ). This gait speed relation with load on cane confirms that the users with poor condition need more assistance than other with lower condition. Volunteer 6 is an outlayer in this analysis because he presents a vestibular disorder, i.e. he uses the cane for balance rather than for weight bearing.

In brief, if our system detects that the load on a cane grows significantly for any given user, it may send a request for assistance and/or a more complete assessment to any nearby telecare robot.

Finally, it can be observed in Figure 6 that the upper bound for our test volunteers is equal to $12 \mathrm{~kg}$. For 8 hours per day of loading, the reported battery duration of three days without charging is comfortably met.

\section{DISCUSSION AND FUTURE WORK}

This paper has presented a low cost modular system detect anomalies in load on cane for elderly people, so that telecare robots may be preemptively warned to provide assistance and/or more exhaustive assessment. The main advantages of the proposed system are its cost and low power consumption. Hence, people can use it for extended time periods whenever and wherever they are walking. Also, our system does not affect the ergonomy nor the centre of gravity of the cane and it can be easily attached to any existing standard one. The system has been embedded into a commercial cane and tested in senior facilities in Cordoba, Andalusia. All volunteers were elderly people that required a cane for everyday mobility. We used a 10 Meters Test for benchmarking to prove that load on cane is related to speed and, hence, their condition. This Test also proves that our system discriminates among volunteers with different conditions.

The main contribution of this work is that our system extends the reading range of cheap sensors up to $30 \mathrm{Kg}$, which is enough for most of our target population (people of different weights with different disabilities that require a cane to walk). The measured error on load on cane estimation is under $0.14 \mathrm{Kg}$, so it can be used to monitor trends and walking behaviours in users and check for condition changes or potential risks. Last but not least, the battery life tests have proven that the system can by used for long-term monitoring (over 3 days without charging).

Our device can be attached to any standard commercial cane in a simple way. All 3D models, plus the microcontroller software are freely available online at https://github.com/joaquinballesteros/Smart-Cane. All the 


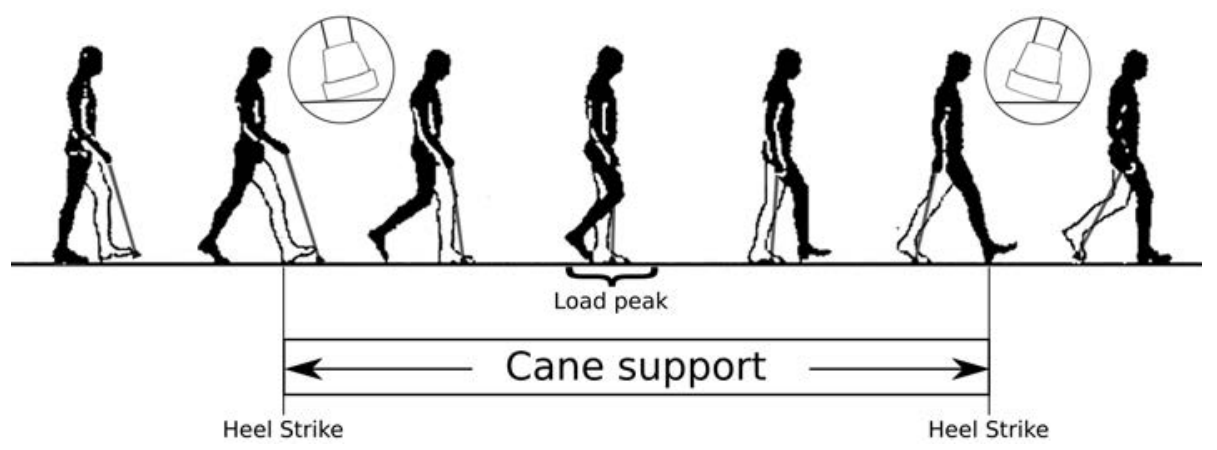

(a)

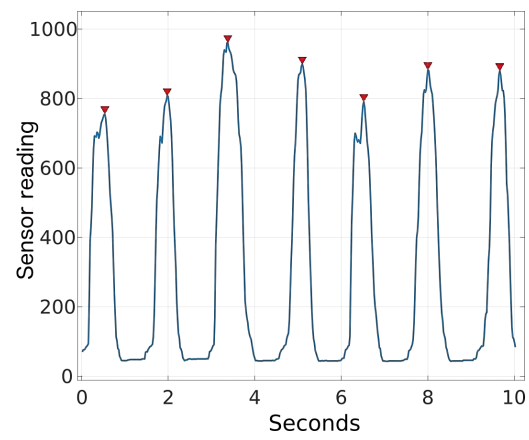

(b)

Fig. 5. Contralateral user walking: (a) User walking in the support cane period; and (b) Input signal from force sensor during a 10 seconds interval in user 8 (range is $0-1023$ ). Maximum peaks (corresponding to cane vertical position) are marked in $\boldsymbol{\nabla}$.

TABLE I

CONDITION AND CHARACTERISTICS PER USERS

\begin{tabular}{|c|c|c|c|c|}
\hline Id & Age & Gender & Gait Speed & Physical issues \\
\hline \hline 1 & 80 & M & $0.615 \mathrm{~m} / \mathrm{s}$ & Visual impairment; osteoarthritis; low back pain \\
\hline 2 & 78 & F & $0.654 \mathrm{~m} / \mathrm{s}$ & Osteoarthritis (right side shoulder and leg); spinal discs herniation \\
\hline 3 & 85 & F & $0.607 \mathrm{~m} / \mathrm{s}$ & Meniscus surgery in both knees \\
\hline 4 & 87 & M & $0.498 \mathrm{~m} / \mathrm{s}$ & Osteoarthritis(left knee) \\
\hline 5 & 86 & M & $0.687 \mathrm{~m} / \mathrm{s}$ & Heart surgery. Lower limbs weakness \\
\hline 6 & 91 & M & $0.597 \mathrm{~m} / \mathrm{s}$ & Vestibular disorder \\
\hline 7 & 76 & M & $0.763 \mathrm{~m} / \mathrm{s}$ & Visual impairment; low back pain \\
\hline 8 & 74 & M & $0.792 \mathrm{~m} / \mathrm{s}$ & Right knee prosthesis \\
\hline
\end{tabular}

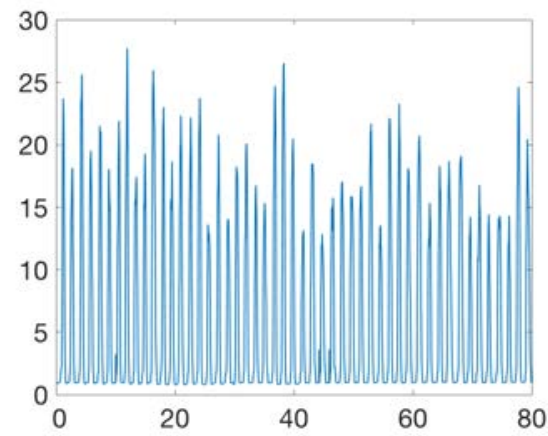

(a) User 4

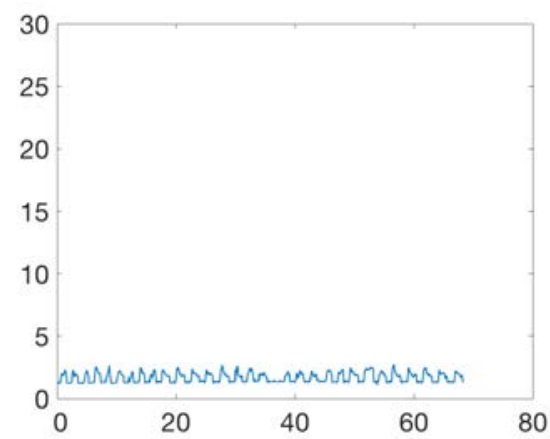

(c) User 5

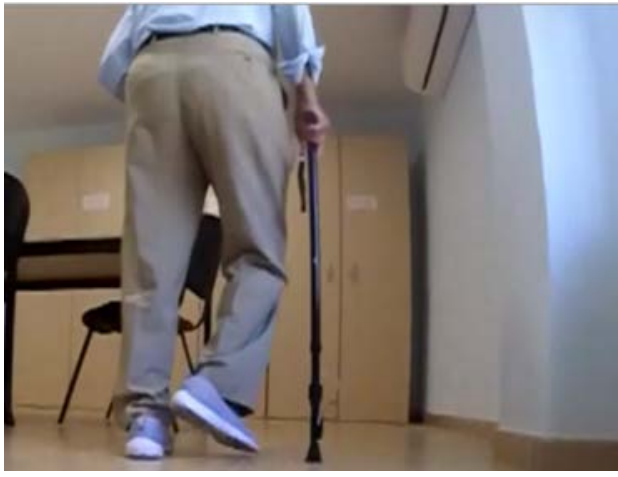

(b) User 4

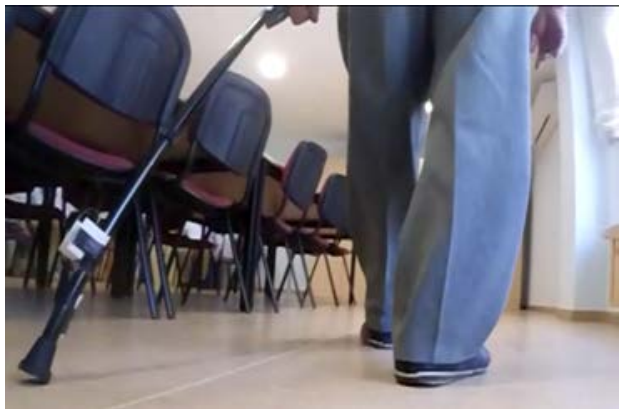

(d) User 5

Fig. 6. Load on cane y-axis (kg) over time x-axis (seconds) 


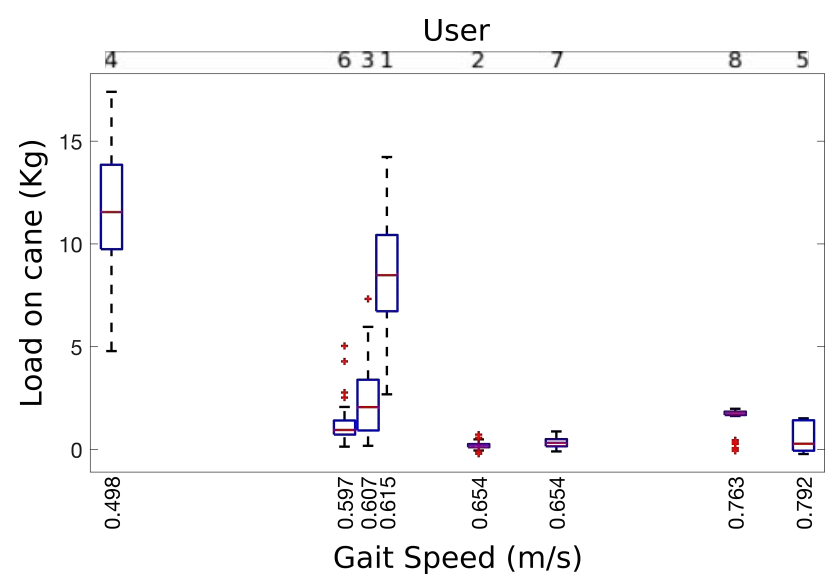

Fig. 7. Load on cane vs users' gait speed

materials are under the terms and conditions of the Creative Commons Attribution 4.0. Its commercial use, modification and/or distribution is allowed for better dissemination and distribution.

Future work will focus on analysing whether the cane can provide more parameters of interest related to users' condition. Also, we plan to develop a ROS package to deploy the proposed sensor system into any telecare robot architecture in a simple way. Then, we plan to use a CGA robot to assess the cane user with known clinic scales to ultimately check the validity of the system as attention mechanism for telecare robots.

\section{REFERENCES}

[1] A. Bandera, J. P. Bandera, P. Bustos, L. V. Calderita, A. Duenas, F. Fernández, R. Fuentetaja, A. Garcia-Olaya, F. J. Garcia-Polo, J. C. González et al., "Clarc: a robotic architecture for comprehensive geriatric assessment," in Proceedings of the 17th Workshop of Physical Agents (WAF), 2016, pp. 1-8.

[2] H. Robinson, B. MacDonald, and E. Broadbent, "The role of healthcare robots for older people at home: A review," International Journal of Social Robotics, vol. 6, no. 4, pp. 575-591, 2014.

[3] S. Jenkins and H. Draper, "Care, monitoring, and companionship: views on care robots from older people and their carers," International Journal of Social Robotics, vol. 7, no. 5, pp. 673-683, 2015.

[4] L. Carcreff, C. N. Gerber, A. Paraschiv-Ionescu, G. De Coulon, C. J. Newman, S. Armand, and K. Aminian, "What is the best configuration of wearable sensors to measure spatiotemporal gait parameters in children with cerebral palsy?" Sensors, vol. 18, no. 2, 2018.

[5] N. M. Gell, R. B. Wallace, A. Z. Lacroix, T. M. Mroz, and K. V. Patel, "Mobility device use in older adults and incidence of falls and worry about falling: Findings from the 2011-2012 national health and aging trends study," Journal of the American Geriatrics Society, vol. 63, no. 5, pp. 853-859, 2015.

[6] J. Wade, M. Beccani, A. Myszka, E. Bekele, P. Valdastri, P. Flemming, M. de Riesthal, T. Withrow, and N. Sarkar, "Design and implementation of an instrumented cane for gait recognition," in Robotics and Automation (ICRA), 2015 IEEE International Conference on. IEEE, 2015, pp. 5904-5909.

[7] R. L. Routson, M. Bailey, I. Pumford, J. M. Czerniecki, and P. M. Aubin, "A smart cane with vibrotactile biofeedback improves cane loading for people with knee osteoarthritis," in Engineering in Medicine and Biology Society (EMBC), 2016 IEEE 38th Annual International Conference of the. IEEE, 2016, pp. 3370-3373.

[8] P. Hellstrom, M. Folke, and M. Ekström, "Wearable weight estimation system,” Procedia Computer Science, vol. 64, pp. 146-152, 2015.
[9] J. Ballesteros, C. Urdiales, A. B. Martinez, and M. Tirado, "Gait analysis for challenged users based on a rollator equipped with force sensors," in Intelligent Robots and Systems (IROS), 2015 IEEE/RSJ International Conference on. IEEE, 2015, pp. 5587-5592.

[10] — "Automatic assessment of a rollator-user's condition during rehabilitation using the i-walker platform," IEEE Transactions on Neural Systems and Rehabilitation Engineering, vol. 25, no. 11, pp. 2009-2017, 2017.

[11] J. Ballesteros, C. Urdiales, A. B. Martinez, and J. H. van Dieën, "On gait analysis estimation errors using force sensors on a smart rollator," Sensors, vol. 16, no. 11, p. 1896, 2016.

[12] S. Hesse, C. Bertelt, M. Jahnke, A. Schaffrin, P. Baake, M. Malezic, and K. Mauritz, "Treadmill training with partial body weight support compared with physiotherapy in nonambulatory hemiparetic patients," Stroke, vol. 26, no. 6, pp. 976-981, 1995.

[13] S. Fritz and M. Lusardi, "White paper:"walking speed: the sixth vital sign"," Journal of geriatric physical therapy, vol. 32, no. 2, pp. 2-5, 2009.

[14] J. Mercado, G. Chu, E. J. Imperial, K. G. Monje, R. M. Pabustan, and A. Silverio, "Smart cane: Instrumentation of a quad cane with audio-feedback monitoring system for partial weight-bearing support," in Bioelectronics and Bioinformatics (ISBB), 2014 IEEE International Symposium on. IEEE, 2014, pp. 1-4.

[15] K. Kumar, B. Champaty, K. Uvanesh, R. Chachan, K. Pal, and A. Anis, "Development of an ultrasonic cane as a navigation aid for the blind people," in Control, Instrumentation, Communication and Computational Technologies (ICCICCT), 2014 International Conference on. IEEE, 2014, pp. 475-479.

[16] M. Rahman, A. Abdullah, A. Mohammad, M. Nordin, M. M. Ali, and M. Saaid, "Smart cane with human detection capabilities for blind people," Journal of Fundamental and Applied Sciences, vol. 10, no. 6S, pp. 989-1004, 2018.

[17] D. C. Dang and Y. S. Suh, "Walking distance estimation using walking canes with inertial sensors," Sensors, vol. 18, no. 1, p. 230, 2018.

[18] D. R. Aragaki, M. C. Nasmyth, S. C. Schultz, G. M. Nguyen, J. M. Yentes, K. Kao, K. Perell, and M. A. Fang, "Immediate effects of contralateral and ipsilateral cane use on normal adult gait," $P M \& R$, vol. 1, no. 3, pp. 208-213, 2009.

[19] J. H. Hollman, E. M. McDade, and R. C. Petersen, "Normative spatiotemporal gait parameters in older adults," Gait \& posture, vol. 34, no. 1, pp. 111-118, 2011. 